\title{
NDRG4, an early detection marker for colorectal cancer, is specifically expressed in enteric neurons
}

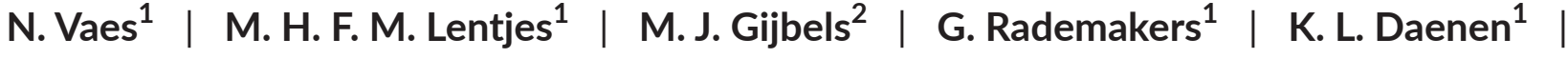 \\ W. Boesmans ${ }^{1,3}$ | K. A. D. Wouters ${ }^{1}$ | A. Geuzens ${ }^{3} \mid$ X. $\mathbf{Q u}^{4} \mid$ H. P. J. Steinbusch ${ }^{5}$ |

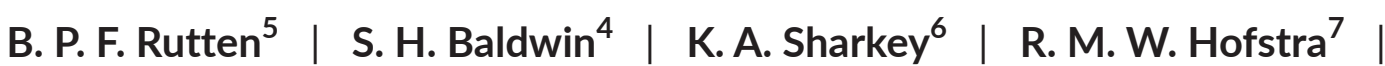 \\ M. van Engeland ${ }^{1}$ | P. Vanden Berghe ${ }^{3}$ | V. Melotte ${ }^{1,7}$ \\ ${ }^{1}$ Department of Pathology, GROW-School for Oncology and Developmental Biology, Maastricht University Medical Center, Maastricht, The Netherlands \\ ${ }^{2}$ Departments of Pathology and Molecular Genetics, Cardiovascular Research Institute Maastricht (CARIM), Maastricht University; Department of Medical \\ Biochemistry, Academic Medical Center, Amsterdam, The Netherlands \\ ${ }^{3}$ Laboratory for Enteric Neuroscience (LENS); Translational Research Center for Gastrointestinal Disorders (TARGID), Department of Clinical and Experimental \\ Medicine, University of Leuven, Leuven, Belgium \\ ${ }^{4}$ Department of Pediatric Cardiology, Vanderbilt University Medical Center, Nashville, TN, USA \\ ${ }^{5}$ Department of Psychiatry and Neuropsychology, School for Mental Health and Neuroscience (MHeNS), Maastricht University Medical Centre, Maastricht, \\ The Netherlands \\ ${ }^{6}$ Hotchkiss Brain Institute and Snyder Institute for Chronic Diseases, Department of Physiology and Pharmacology, Cumming School of Medicine, University of \\ Calgary, Calgary, Alberta, Canada \\ ${ }^{7}$ Department of Clinical Genetics, University of Rotterdam, EMC, Rotterdam, The Netherlands
}

\section{Correspondence}

Veerle Melotte, Department of Pathology, GROW - School for Oncology and Developmental Biology, Maastricht University Medical Center, Maastricht, The Netherlands. Email: veerle.melotte@maastrichtuniversity.nl

Funding Information

This work was supported by a KWF Kankerbestrijding grant (UM 2013-6075) obtained by Dr. Veerle Melotte.

\begin{abstract}
Background: Promoter methylation of N-myc Downstream-Regulated Gene 4 (NDRG4) in fecal DNA is an established early detection marker for colorectal cancer (CRC). Despite its connection to CRC, NDRG4 is predominantly studied in brain and heart, with little to no knowledge about its expression or role in other organs. In this study, we aimed to determine the whole-body expression of NDRG4, with a focus on the intestinal tract.

Methods: We investigated NDRG4 expression throughout the body by immunohistochemistry, Western Blotting and in situ mRNA hybridization using tissues from NDRG4 wild-type, heterozygous and knockout mice and humans. In addition, we explored cellspecific expression of NDRG4 in murine whole-mount gut preparations using immunofluorescence and confocal microscopy.

Key Results: NDRG4 is specifically expressed within nervous system structures throughout the body. In the intestinal tract of both mouse and man, NDRG4 immunoreactivity was restricted to the enteric nervous system (ENS), where it labeled cell bodies of the myenteric and submucosal plexuses and interconnecting nerve fibers. More precisely, NDRG4 expression was limited to neurons, as NDRG4 always colocalized with HuC/D (pan-neuronal marker) but never with GFAP (an enteric glial cell
\end{abstract}


marker). Furthermore, NDRG4 was expressed in various neuropeptide $Y$ positive neurons, but was only found in a minority ( 10\%) of neurons expressing neuronal nitric oxide synthase.

Conclusions and Inferences: NDRG4 is exclusively expressed by central, peripheral and enteric neurons/nerves, suggesting a neuronal-specific role of this protein. Our findings raise the question whether NDRG4, via the ENS, an understudied component of the tumor microenvironment, supports CRC development and/or progression.

KEYWORDS

biomarker, colorectal cancer, enteric nervous system, myenteric plexus, NDRG4

\section{1 | INTRODUCTION}

Previously, we identified N-myc Downstream-Regulated Gene 4 (NDRG4) promoter $\mathrm{CpG}$ island methylation as a promising early detection marker for colorectal cancer (CRC), ${ }^{1}$ an observation that has been independently validated. ${ }^{2,3}$ NDRG4 is one of the molecular markers of the FDAapproved, multi-target stool DNA test $\left(\right.$ Cologuard $^{\circledR}$, Exact Sciences Corporation, Madison, USA), which detects significantly more cancers than the leading fecal immunochemical test (FIT), and is currently used as a screening modality in the USA. ${ }^{3}$ Surprisingly, given its connection to $C R C$, the expression pattern and function(s) of NDRG4 in the gut and its role in CRC carcinogenesis remain to be elucidated.

NDRG4, also known as SMAP-8 and BDM1, is one of the four members (NDRG1-4) of the NDRG gene family, a group of genes involved in cell proliferation, differentiation, development and stress. ${ }^{4}$ The encoded proteins of this family, characterized by an $\alpha / \beta$ hydrolase fold, share $52 \%-65 \%$ sequence homology and are highly conserved in evolution in various species. ${ }^{5,6}$ The NDRG4 gene was originally characterized in humans and encodes three isoforms: NDRG4H (41 kDa), NDRG4B (37 kDa) and NDRG4B ${ }^{\mathrm{var}}(39 \mathrm{kDa}){ }^{5}$ Although all three human isoforms have been identified in mouse and rat brain, the latter reveals an additional fourth isoform $(45 \mathrm{kDa})$ encoded by the rat ortholog of human NDRG4: 'SMAP-8/BDM1', while the mouse orthologs correspond to NDRG4B and NDRG4B ${ }^{\text {var } 7,8}$ In contrast to the well-described ubiquitous expression of NDRG1, NDRG2 and NDRG3, abundant expression of NDRG4 is predominantly observed in brain and heart, suggesting an important role of NDRG4 in these organs. ${ }^{5-10}$ In fact, in the central nervous system (CNS), NDRG4 is required for normal morphogenesis of the mouse and zebrafish brain and to protect against neurological deficits by maintaining physiological levels of brain-derived neurotrophic factor (BDNF). ${ }^{7,10}$ NDRG4 is essential for proper neurite outgrowth, neural functions in vitro and myelination of axons in zebrafish. ${ }^{11,12}$ Consistent with these observations, the Alzheimer diseased brain, which is characterized by neuronal degeneration, shows reduced levels of NDRG4. ${ }^{5}$ Furthermore, proper morphogenesis of the mouse and zebrafish heart is regulated by NDRG4 and NDRG4 deficiency is associated with weak contractility and reduced heart rate in zebrafish. ${ }^{10,13}$ Similarly, variations in locus 16q21 near NDRG4 have been associated with prolongation of the QT interval, a risk factor for sudden cardiac death. ${ }^{14}$

\section{KEY POINTS}

- Despite its excellent performance as biomarker, the expression and function of NDRG4 in the gut and its role in CRC carcinogenesis are unknown

- NDRG4 is specifically expressed in neurons of the enteric nervous system

- The specific expression of NDRG4 unravels a novel neuronspecific role of this protein and opens the discussion of whether the ENS contributes to CRC carcinogenesis

Nevertheless, the molecular mechanisms by which NDRG4 affects the above-described phenomena are still unknown.

As the expression and functional role(s) of NDRG4 outside the heart and brain are largely unstudied, we examined the overall wholebody expression of NDRG4, while focusing on the intestinal tract as a first step in understanding the role(s) of NDRG4 in the gut. Therefore, we determined NDRG4 expression according the two main specificity criteria as described by Pradidarcheep et al., ${ }^{15}$ by means of immunohistochemistry, in situ mRNA hybridization and Western blotting using human, NDRG4 wild-type and NDRG4 knockout mouse tissues. In addition, we investigated cell-specific expression of NDRG4 in more depth in murine whole-mount gut preparations using immunofluorescence.

\section{MATERIALS AND METHODS}

\section{1 | Human}

Formalin-fixed, paraffin-embedded human normal colon tissues of CRC patients $(n=5)$ were retrospectively collected from the archive of the Department of Pathology of the Maastricht University Medical Center. Written informed consent was obtained from all study participants and the process adhered to local ethics guidelines.

\section{2 | Mice}

NDRG4 wild-type (NDRG4 $\left.4^{+/+}\right)$, heterozygous $\left(\mathrm{NDRG}^{+/-}\right)$and knock-out $\left(\mathrm{NDRG}^{-/-}\right.$) mice (C57BL/6 genetic background) were kindly provided 
by Prof. Baldwin (Vanderbilt University Medical Center) ${ }^{16}$ and characterized by genotyping PCR (See "Genotyping"). Animals were age- and gender matched and housed in groups of 3-5 under standard conditions having free access to food and water. One-year old NDRG4 $4^{+/+}$, $\mathrm{NDRG}^{+/-}$and NDRG4 $4^{-/-}$mice were sacrificed, tissues were harvested and either snap-frozen for protein isolation or fixed in $4 \%$ formaldehyde for immunohistochemistry. In addition, 2 month old NDRG4 $4^{+/+}$ and NDRG ${ }^{-/-}$mice were sacrificed, followed by removal of brain and intestines for immunostainings on brain and whole-mount gut preparations. ${ }^{17}$ Animal experiments were approved by the Committee of Animal Welfare of Maastricht University and performed according to Dutch regulations.

\section{3 | Genotyping}

To identify carriers of the NDRG4 wild-type and deletion allele, purified DNA was examined by PCR. Genomic DNA was purified with the Gentra Puregene Mouse tail kit (Qiagen, Maryland, USA) according to manufacturer's instructions. For the detection of each allele, a PCR was performed with the NDRG4 primer mix listed in Table 1. The PCR reaction mix contained $100 \mathrm{ng}$ genomic DNA, $10 \mu \mathrm{L}$ REDExtract-NAmp PCR Reaction Mix (REDExtract-N-Amp ${ }^{\text {TM }}$ Tissue PCR Kit, SigmaAldrich, St. Louis, MO, USA) and $0.8 \mu \mathrm{L}$ primer mix $(10 \mu \mathrm{M})$ in a final volume of $20 \mu \mathrm{L}$. The PCR was performed using the Biorad T100 ${ }^{\mathrm{TM}}$ thermal cycler (Biorad) with the following conditions: (i) initial incubation: $94^{\circ} \mathrm{C}$ for 3 minutes, (ii) 35 cycles: $94^{\circ} \mathrm{C}$ for 30 seconds $-60^{\circ} \mathrm{C}$ (annealing temperature) for 30 seconds $-72^{\circ} \mathrm{C}$ for 1 minutes, and (iii) final elongation: $72^{\circ} \mathrm{C}$ for 10 minutes. PCR products were detected in a $1.5 \%(\mathrm{w} / \mathrm{v})$ agarose gel in $0.5 \times$ Tris-borate-EDTA (TBE) buffer. The NDRG4 wild-type locus is identified by a 203 and 957 bp fragment, whereas a $320 \mathrm{bp}$ band identifies the deletion allele.

\subsection{Protein isolation and western blotting}

Brain, heart and colon tissues from NDRG4 $4^{+/+}, \mathrm{NDRG}_{4}^{+/-}$and NDRG4 $4^{-/-}$ mice were homogenized in RIPA buffer (Pierce Technology, Rockford, Illinois, USA) containing protease inhibitors (1 "complete" pill/50 mL, Roche, Mannheim, Germany), and resolved in SDS-gel electrophoresis. Protein transfers were probed overnight $\left(4^{\circ} \mathrm{C}\right)$ with mouse anti-human NDRG4, clone 2G3 (1:500; H00065009-M01; Abnova, Taipei City, Taiwan), rabbit anti-human NDRG4 (1:1000; \#9039; Cell Signaling, Leiden, The Netherlands) and $\beta$-actin (1:200 000; SigmaAldrich). Bound antibodies were visualized by an HRP-linked secondary anti-rabbit or anti-mouse antibody (1 hour, RT, Cell Signaling) and chemiluminescence (ECL, Pierce Technology, Rockford, Illinois, USA).

\section{5 | Immunohistochemistry}

Three $\mu \mathrm{m}$ thick paraffin sections of every organ of $\mathrm{NDRG}^{+/+}$and $N D R G 4^{-/-}$mice and human colon were deparaffinized in xylene and rehydrated in graded alcohols. To quench endogenous peroxidase activity, the slides were incubated with $0.3 \%$ hydrogen peroxide in methanol for 20 minutes. Antigen retrieval was performed by boiling the sections in Tris-EDTA buffer (pH 8.0; Klinipath, Duiven, The Netherlands) or Dako target retrieval solution ( $\mathrm{pH}$ 6.0; Dako, Santa Clara, CA, USA), followed by blocking nonspecific antibody binding with PBS containing $20 \%$ fetal bovine serum and $0.1 \%$ Tween. Sections where incubated overnight at $4{ }^{\circ} \mathrm{C}$ with the primary antibodies: mouse anti-human NDRG4, clone 2G3 (1:500, Abnova) or rabbit anti-human NDRG4 antibody (Mouse brain and other organs 1:500 and 1:250, resp.; Human colon, 1:25; Cell Signaling) diluted in PBS/0.5\%BSA/0.1\%Tween. After incubation with the biotinylated anti-mouse secondary antibody (1:250; RPN1001v1; Amerscham Biosciences, Piscataway, NJ, USA) followed by the Avidin-Biotin Complex detection method (ABC; 1:500; Dako Cytomation, Glostrup, Denmark) or incubation with horseradish peroxidase-conjugated anti-rabbit IgGs (poly-HRP, Immunologic, Duiven, The Netherlands), bound antibodies were visualized using 3,3'-diaminobenzidine (DAB, Dako) as a chromogen (brown precipitate). Slides were counterstained with hematoxylin, dehydrated and mounted. In addition, to diminish recognition of endogenous mouse immunoglobins by the mouse primary antibody, slides were subsequently stained with the mouse anti-human NDRG4 antibody, clone $2 \mathrm{G} 3$ (1:500; H00065009-M01; Abnova) using the Vector ${ }^{\circledR}$ M.O.M. ${ }^{\mathrm{TM}}$ Immunodetection Kit according to manufacturer's instructions (Vector Labs, Burlingame, CA, USA). Negative controls for primary antibodies were monitored by staining of NDRG $4^{-/-}$tissues and omission of primary antibodies from NDRG4 $4^{+/+}$slides. Images were acquired at RT using a Nikon DMX1200 digital camera and the ACT-1 v2.62 software from Nikon Corporation (Amsterdam, The Netherlands).

\section{6 | Immunofluorescence}

Immunostainings were performed on brain slices and whole-mount gut preparations as previously described. ${ }^{17}$ Briefly, ileum and colon of $N D R G 4^{+/+}$and NDRG4 ${ }^{-/-}$mice were collected, opened along the mesenteric border, stretched and pinned flat with insect pins $(0.2 \mathrm{~mm}$, Agar Scientific, Stansted, UK) in a Sylgard-lined dissection dish (Sylgard 184 Elastomer, Down Corning, Auburn, MI, USA) with Krebs solution bubbled with $95 \% \mathrm{O}_{2}-5 \% \mathrm{CO}_{2}$. The mucosal and submucosal layers were removed, tissues fixed for 30 minutes $\left(4^{\circ} \mathrm{C}\right)$ in paraformaldehyde (4\% in PBS) and rinsed in PBS. To visualize the myenteric and

TABLE 1 PCR primers for genotyping

\begin{tabular}{|cll}
\hline Target & Forward primer & Reverse primer \\
\hline NDRG4 mouse & & \\
\hline NDRG4 4HLOX1 & TAGGCAGGGGCAGGTGGGTTTGT & \\
\hline NDRG4 4HLOX2 & & GGCGTCCTGATGTCATGTTCCTGT \\
\hline NDRG4 4H776 & & GCTCCCACTCCAATGCCAATC
\end{tabular}


submucosal plexus the circular or longitudinal muscle layer, respectively, were peeled. Brain and gut tissues were permeabilized in $0.5 \%$ tritonX-100 and incubated in blocking solution (4\% goat/donkey serum), followed by an overnight incubation $\left(4^{\circ} \mathrm{C}\right)$ with the primary antibodies diluted in blocking solution: rabbit anti-NDRG4 (1:500, Cell Signaling), chicken anti-GFAP (1:5000; Abcam, Cambridge, UK), human anti-HuC/HuD (1:500; Invitrogen Life Technologies, Rockford, Illinois, USA), sheep anti-neuronal NO-synthase (1:400; Santa Cruz Biotechnologies, Delaware, CA, USA), rabbit anti-calretinin (1:2000; Chemicon, Darmstadt, Germany), rat anti-neuropeptide $Y$ (1:500; Eugene Tech International Allendale, NJ, USA) for whole-mount gut preparations; rabbit anti-NDRG4 (1:500; Cell Signaling) and mouse anti-human NeuN (1:100, Chemicon) for brain slides. After rinsing, tissues were incubated for 2 hours with the fluorescently labeled secondary antibodies: anti-human Alexa594, anti-mouse Alexa594, anti-rabbit Alexa488, anti-sheep Alexa488, anti-rat Alexa488 (all 1:1000; Molecular probes, Invitrogen, Belgium), anti-rabbit AMCA or anti-chicken AMCA (both 1:250; Jackson Immuno Research Labs, West Grove, PA, USA). Samples were mounted with Vectashield ${ }^{\text {TM }}$ mounting medium (Vector Labs, Burlingame, CA, USA). Preparations were imaged with a Zeiss LSM780 confocal microscope (Cell imaging Core, KU Leuven).

\section{7 | In situ mRNA hybridization}

In situ mRNA hybridization was performed on paraffin-embedded brains and intestinal Swiss rolls of NDRG4 ${ }^{+/+}$and NDRG4 ${ }^{-/-}$mice using digoxigenin (DIG) labeled riboprobes (DIG RNA Labeling Kit, SP6/T7, Roche) for NDRG4 nucleotide positions 1269-1777 (NDRG4-A) and 1811-2343 (NDRG4-B) of mouse NDRG4 (NM_145602, Table 2). ${ }^{7}$ Four $\mu \mathrm{m}$ sections were dried upright, deparaffinized, rehydrated and washed. Tissues were permeabilized with $0.1 \%$ pepsin in $0.2 \mathrm{~N} \mathrm{HCl}$ for 5 minutes $\left(37^{\circ} \mathrm{C}\right)$ and post-fixed in $4 \%$ paraformaldehyde $\left(4^{\circ} \mathrm{C}\right)$. Fixed sections were then treated twice with $100 \mathrm{mM}$ glycine in 1xPBS and prehybridized $\left(37^{\circ} \mathrm{C}\right)$ for 45 minutes in prehybridization buffer $(2 \times$ SCC, $50 \%(v / v)$ deionized formamide, 40\% (v/v) DEPC-treated $\mathrm{H}_{2} \mathrm{O}$ ). NDRG4 mRNA was detected overnight at $37^{\circ} \mathrm{C}$ with the preheated hybridization buffer $(2 \times$ SCC, $50 \%(\mathrm{v} / \mathrm{v})$ formamide, $10 \%$ dextran sulphate, $10 \mathrm{mM}$ DTT,7,5\% DEPC- $\mathrm{H}_{2} \mathrm{O}, 1 \times$ Denhardts solution, $1 \mathrm{mg} /$ $\mathrm{mL}$ yeast tRNA, $1 \mathrm{mg} / \mathrm{mL}$ denatured and sheared salmon sperm DNA) containing a mixture of both antisense or sense probes $(1 \mu \mathrm{g} / \mathrm{mL})$. After a serial wash in descending concentrations $(2 \times, 1 \times$ and $0.1 \times)$ of SCC buffer, tissue RNA was digested by RNase A (Roche), followed by a serial wash in ascending SCC concentrations ( $1 \times$ and $2 \times)$ and buffer 1 (100 mM Tris- $\mathrm{HCl}, 150 \mathrm{mM} \mathrm{NaCl}$ ). Sections were then blocked for two hours (0.1\% Triton X-100, 2\% normal sheep serum in buffer 1 ) and incubated with 1:100 anti-DIG alkaline phosphatase Fab fragments (Roche) for 1 hour at $37^{\circ} \mathrm{C}$. Following washes in buffer 1 and $2(100 \mathrm{mM}$ Tris- $\mathrm{HCl}, 100 \mathrm{mM} \mathrm{NaCl}, 50 \mathrm{mM} \mathrm{MgCl}$ ), the chromogenic substrates nitro-blue tetrazolium chloride and 5-bromo-4chloro-3indolyl phosphate (Roche), dissolved in buffer 2 containing $5 \mathrm{mM}$ levamisole, were used to detect hybridized alkaline phosphatase activity. Once the signal reached optimal intensity, the color reaction was stopped (10 mM Tris- $\mathrm{HCl}, 1 \mathrm{mM}$ EDTA), sections were counterstained with nuclear fast red, clarified in tap water and mounted in imsol (1:3). Tissue sections of NDRG4 $4^{-/-}$and NDRG4 ${ }^{+/+}$mice immersed in antisense or sense riboprobes, respectively, served as negative controls. Images were acquired as described for IHC images.

\section{3 | RESULTS}

\section{1 | The Cell Signaling antibody is able to specifically target NDRG4}

We first characterized the specificity of both anti-NDRG4 antibodies using tissues of $\mathrm{NDRG}^{+/+}, \mathrm{NDRG}^{+/-}$and $\mathrm{NDRG}^{-/-}$mice in Western Blotting and immunohistochemistry (Figure 1). Western Blotting revealed that both antibodies could discriminate all known NDRG4 isoforms in various tissues, including proximal and distal colon of $\mathrm{NDRG}^{+/+}$and NDRG4 ${ }^{+/-}$mice (Figure $1 \mathrm{~A}, \mathrm{~B}$ ). However, successful deletion of NDRG4 in NDRG4 ${ }^{-/-}$mice was only confirmed after application of the Cell Signaling antibody. The specificity of the Abnova antibody, however, could not be confirmed, as the signal was still detectable in $\mathrm{NDRG}^{-/-}$tissues and several non-specific bands of about 50-55 kDa were recognized. Non-specific recognition of NDRG4 by the Abnova antibody was also confirmed by the similar staining pattern observed in all examined tissues of $N D R G 4^{-/-}$and $\mathrm{NDRG}^{+/+}$mice (Figure $1 \mathrm{C}$ ). The Cell Signaling antibody on the other hand, detected NDRG4 in $\mathrm{NDRG}_{4}^{+/+}$, but not in NDRG4 $4^{-/-}$tissues (Figure 1D). Thus, only the Cell Signaling antibody (\#9039) is able to specifically target NDRG4 and was therefore further used to comprehensively study the expression of NDRG4 throughout the body and specifically within the gut.

\subsection{NDRG4 is specifically expressed within the enteric nervous system of both mouse and man}

Characterization of NDRG4 expression in the gastrointestinal (GI) tract (ie, stomach, small and large intestine) of $\mathrm{NDRG}_{4}^{+/+}$and $\mathrm{NDRG}^{-/-}$ mice using immunohistochemistry, revealed that NDRG4 is specifically expressed within the nervous system of the gut, ie, the enteric nervous system (ENS; Figure 2). ${ }^{18}$ More precisely, NDRG4 is expressed in the cytoplasm of cell bodies inside ganglia of the myenteric plexus,

TABLE 2 Probes for in situ mRNA hybridization

\begin{tabular}{lll} 
Target & Forward primer & Reverse primer \\
\hline NDRG4 mouse & & \\
NDRG4-A & TTATTTAAAAAGAAATGAGGGGATC & TTGCCTCAGGGTGGGACAA \\
NDRG4-B & GTTAAAATGTTGATTGCTGTGTATGC & ACTCCAGAGCAGTCTAGAAATGGC \\
\hline
\end{tabular}


(A)

\begin{tabular}{|llll|}
\hline \multicolumn{4}{|c|}{ Abnova antibody } \\
\hline Brain & Heart $\quad$ Colon P & Colon D \\
\hline
\end{tabular}

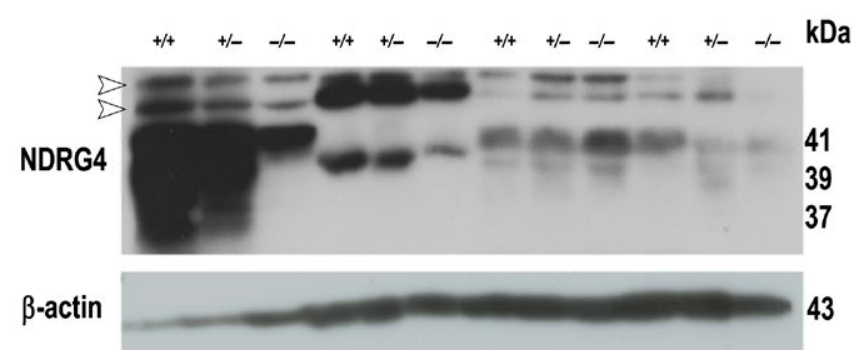

(C)
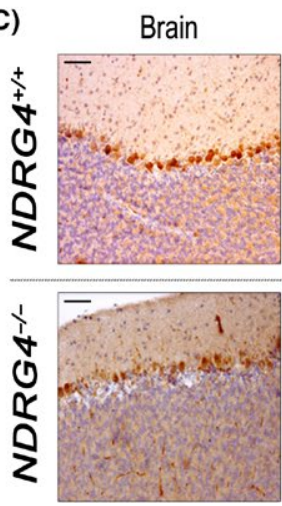

Heart
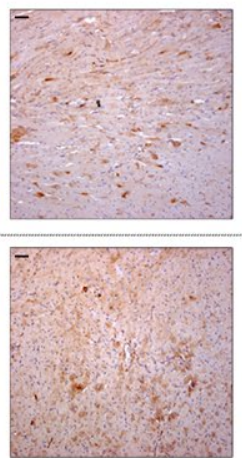

Colon
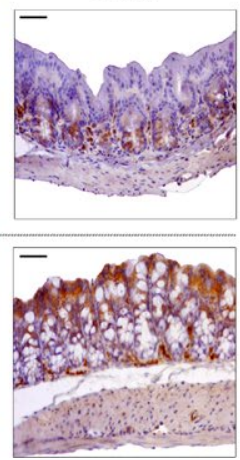

(B)

Cell signaling antibody

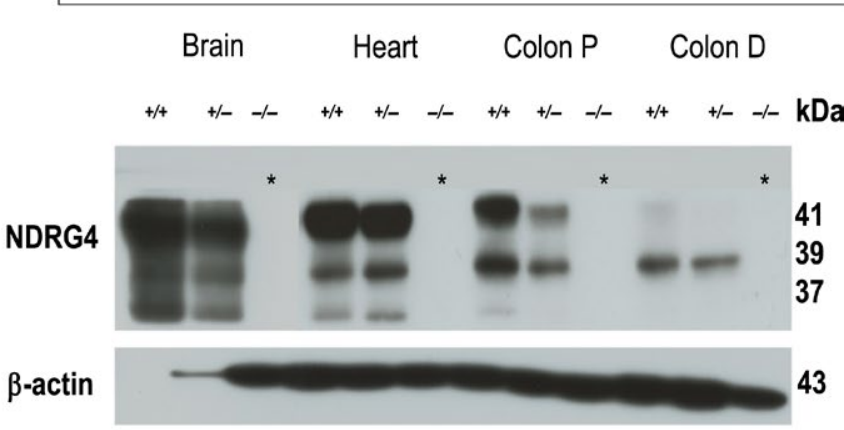

(D)
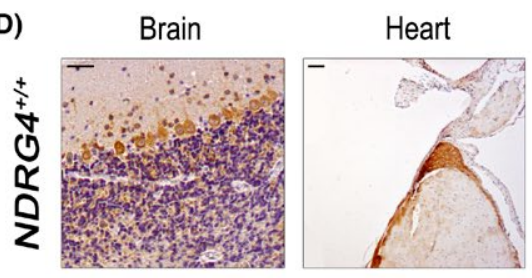

Colon
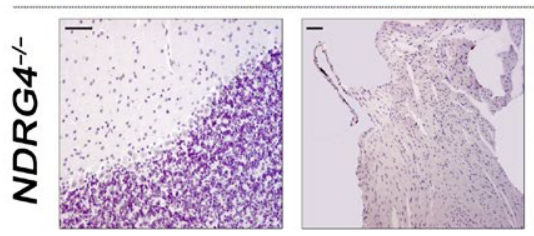

FIGURE 1 The Cell Signaling, but not the Abnova antibody specifically targets NDRG4. (A,B) Western Blotting analysis of NDRG4 ${ }^{+/}$, NDRG4 ${ }^{+/}$ and NDRG4 ${ }^{-/-}$brain, heart and colon (P, proximal; D, distal) using the Abnova (A) and Cell Signaling antibody (B). Both antibodies recognize all three well-known isoforms: NDRG4B (37 kDa), NDRG4B ${ }^{\mathrm{var}}(39 \mathrm{kDa})$ and NDRG4H (41 kDa) in NDRG4 ${ }^{+/+}$and NDRG4 ${ }^{+/-}$tissues. Open triangles indicate non-specific bands (50-55 kDa) identified by the Abnova antibody (A). * indicates successful detection of NDRG4 ablation only after application of the Cell Signaling antibody (B). $\beta$-actin is used as loading control. (C,D) Brain, heart and colon sections from 1-year old NDRG4 ${ }^{+/+}$ and NDRG4 ${ }^{-/-}$mice were subjected to IHC with the Abnova (C) and Cell Signaling antibody (D). Positive signals (brown) detected by the Abnova antibody are not affected by absence of NDRG4 and differ from the highly selective staining pattern produced by the Cell Signaling antibody. Scale bars, $50 \mu \mathrm{m}$ in $\mathrm{C}$ and $\mathrm{D}$

located between the outer longitudinal and inner circular muscle layer along the entire $\mathrm{Gl}$ tract and within the ganglia of the submucosal plexus (Figure 2A). The nerve fiber bundles connecting the ganglia in both plexuses also showed NDRG4 expression. Furthermore, the nerves innervating the outer and inner muscularis externa, muscularis mucosae and those projecting into the mucosa also showed NDRG4 immunoreactivity. As explained above, specificity of the antibody was monitored by NDRG4 immunohistochemistry on the gut of NDRG4 ${ }^{-/-}$ mice. Representative immunohistochemical images (Figure 2A) show that the intestinal tract of NDRG4 ${ }^{-/-}$mice still contains all the ENS structures described above, ie, ganglia of the myenteric and submucosal plexus and interconnecting nerve fibers, but does not display NDRG4 immunoreactivity, confirming the absence of NDRG4 expression. Furthermore, in situ mRNA hybridization confirmed that NDRG4 is specifically expressed within the ENS of NDRG4 $4^{+/+}$mice, but not in $\mathrm{NDRG}^{-/-}$mice (Figure 2A). Similar to that described in mouse, human intestinal specimens displayed NDRG4 immunoreactivity in the ganglia of both plexuses, in nerve fibers connecting these ganglia and within the fibers innervating both muscle layers of the muscularis externa and muscularis mucosae, as shown in Figure 2B. Compared to mouse, the mucosal projecting nerve fibers showed more robust
NDRG4 immunoreactivity. Together these data indicate that NDRG4 is specifically expressed within the ENS of mice and humans.

\subsection{NDRG4 is expressed within enteric neuronal cells}

Whole-mount preparations of the submucosal and myenteric plexuses of the $\mathrm{NDRG}^{+/+}$and NDRG4 $4^{-/-}$small and large intestine were used to explore what types of enteric neurons express NDRG4. Since similar results were observed in above-mentioned whole-mount preparations, only data from the colonic myenteric plexus are shown (Figure 3). Immunofluorescent triple labeling revealed that within the gut of $N D R G 4^{+/+}$mice, NDRG4 is exclusively expressed within the cytoplasm of enteric neurons, as NDRG4 positive cells were always labeled for the pan-neuronal marker HuC/D, but never co-expressed the glial cell marker GFAP (Figure 3A). Even though NDRG4 localized in enteric neurons throughout all areas of the ENS, not all enteric neurons (HuC/D positive) expressed NDRG4 (Figure 3A). To further investigate visual differences in the organization of the plexuses between $\mathrm{NDRG}^{+/+}$and $\mathrm{NDRG}^{-/-}$mice, the two main neuronal subsets, positive for the neuronal markers nitric oxide synthase (nNOS; inhibitory 


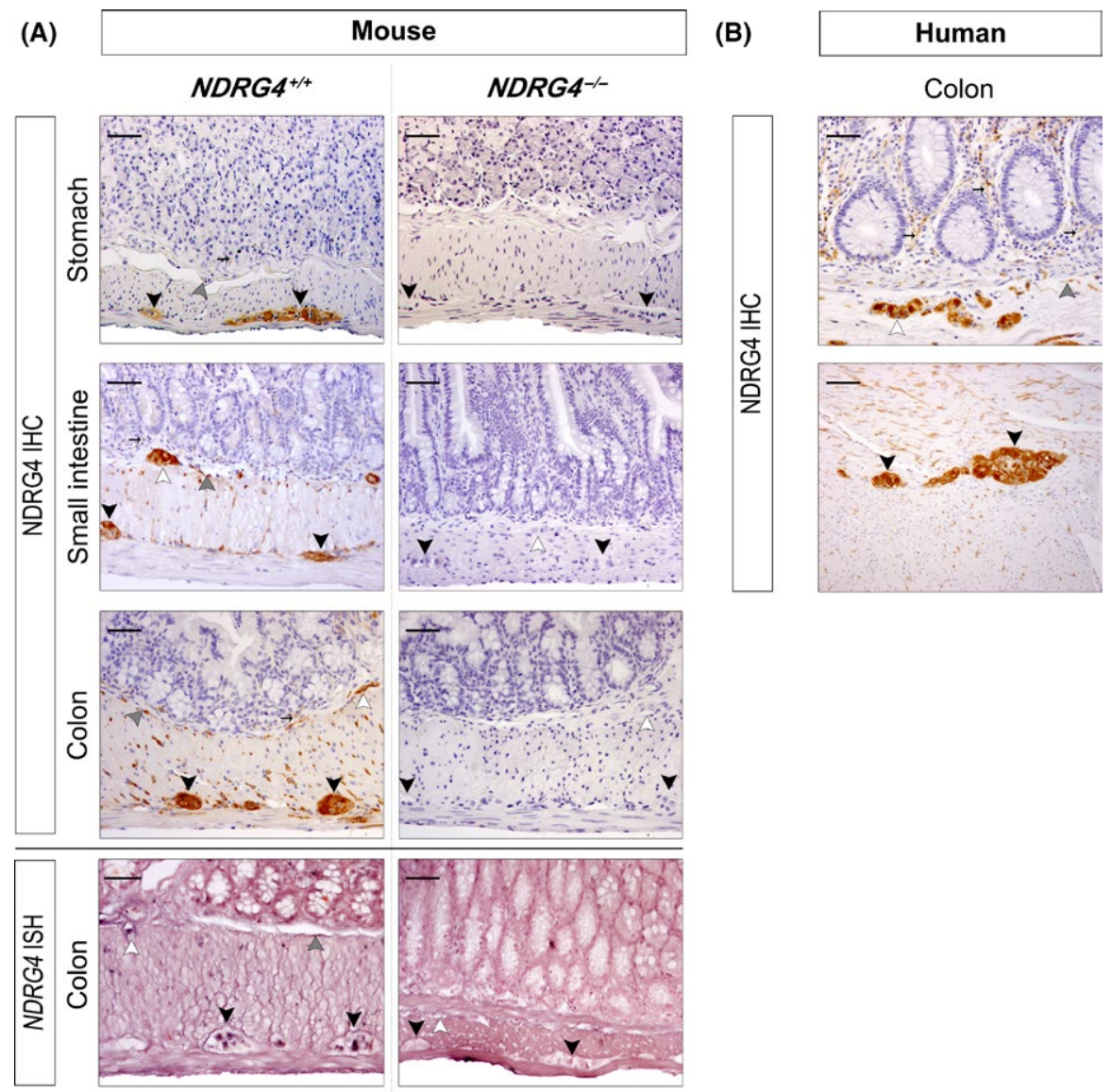

FIGURE 2 NDRG4 is localized within the ENS of mouse and man. A, Representative immunohistochemical staining (IHC) of the GI tract (ie, stomach, small intestine (duodenum) and colon) and colonic in situ mRNA hybridization (ISH) of NDRG4 $4^{+/+}$and $\mathrm{NDRG}^{-/-}$mice. NDRG4 RNA and protein is expressed in myenteric and submucosal ganglia (black and open arrowhead, respectively.), within interconnecting nerve fibers throughout the plexus, muscularis externa, muscularis mucosae (gray arrowhead) and mucosae (black arrow). Deletion of NDRG4 is confirmed at protein and RNA level. B, NDRG4 expression in human colonic specimens is analogous to mouse, with more robustly stained mucosal projecting nerve fibers (black arrows). Scale bars, $50 \mu \mathrm{m}$ in $\mathrm{A}$; and $100 \mu \mathrm{m}$ in $\mathrm{B}$

motor neurons and descending interneurons) or calretinin (excitatory motor neurons), were studied in combination with $\mathrm{HuC} / \mathrm{D}$ (Figure 3B). The overall neuronal population in NDRG4 $4^{+/+}$and NDRG4 ${ }^{-/-}$colon is fairly similar as indicated by analogous HuC/D positivity and calretinin and nNOS staining (Figure 3B). Nevertheless, our preliminary observations reveal that the NDRG4 and nNOS positive population were almost completely different from each other (Figure $3 \mathrm{C}$ ). More precisely, neurons that express NDRG4 are almost exclusively nNOS negative: only $3 \%$ of the NDRG4 positive enteric neurons also expressed nNOS and vice versa, only $10 \%$ of the nNOS population was immunoreactive for NDRG4. Finally, to investigate if NDRG4 is expressed by secretomotor neurons, the co-localization of NDRG4 with the secretomotor neuron marker NPY was studied. Overlap of NDRG4 with NPY was observed in neuronal cell bodies of the intestinal plexuses of $\mathrm{NDRG}^{+/+}$ mice (Figure 3D). In conclusion, NDRG4 is expressed in different subsets of enteric neurons.

\subsection{NDRG4 is expressed by neuronal cells in the central and peripheral nervous system}

Finally, we confirmed that NDRG4 is specifically expressed within the central nervous system (Figure 4A). Neurons in the gray matter structures of the cerebrum (eg, hippocampus and thalamus), cerebellum (eg, Purkinje and molecular layer) and spinal cord of NDRG4 ${ }^{+/+}$mice showed specific, predominant cytoplasmic expression of NDRG4, while the white matter, ependymal cells and meninges were negative for NDRG4. In the peripheral nervous system, we observed a similar staining pattern (Figure 4B). More specifically, the cytoplasm of ganglia situated in close proximity of every organ, ie, prevertebral ganglia, and of the ventral and dorsal root ganglia showed strong NDRG4 reactivity. Furthermore, the tiny nerve fibers throughout peripheral organs itself (eg, in the bronchial branch) also showed strong NDRG4 immunoreactivity. Hence, NDRG4 is almost exclusively expressed in the central, peripheral and enteric nervous system.

\section{4 | DISCUSSION}

Our previous work has shown that the biomarker potential of NDRG4 is associated with a putative tumor suppressor role in vitro. ${ }^{1}$ In addition, we and various independent groups, described that NDRG4 expression in the intestinal epithelium decreases in the transition towards CRC. ${ }^{1,19,20}$ However, all these studies used the commercially available monoclonal mouse anti-human NDRG4 antibody from Abnova (clone 2G3, H00065009-M01), which has, to our knowledge, never been validated to specifically target NDRG4. In this study, we investigated the whole-body expression of NDRG4, focusing on the GI tract, using two commercially available antibodies: the monoclonal mouse anti-human NDRG4 from Abnova (clone 2G3, H00065009-M01) 1,17,20,21 and polyclonal rabbit anti-human NDRG4 from Cell Signaling (\#9039) ${ }^{22,23}$ according to the specificity criteria, ${ }^{15}$ as a critical first step in understanding the role(s) of NDRG4. 
(A)
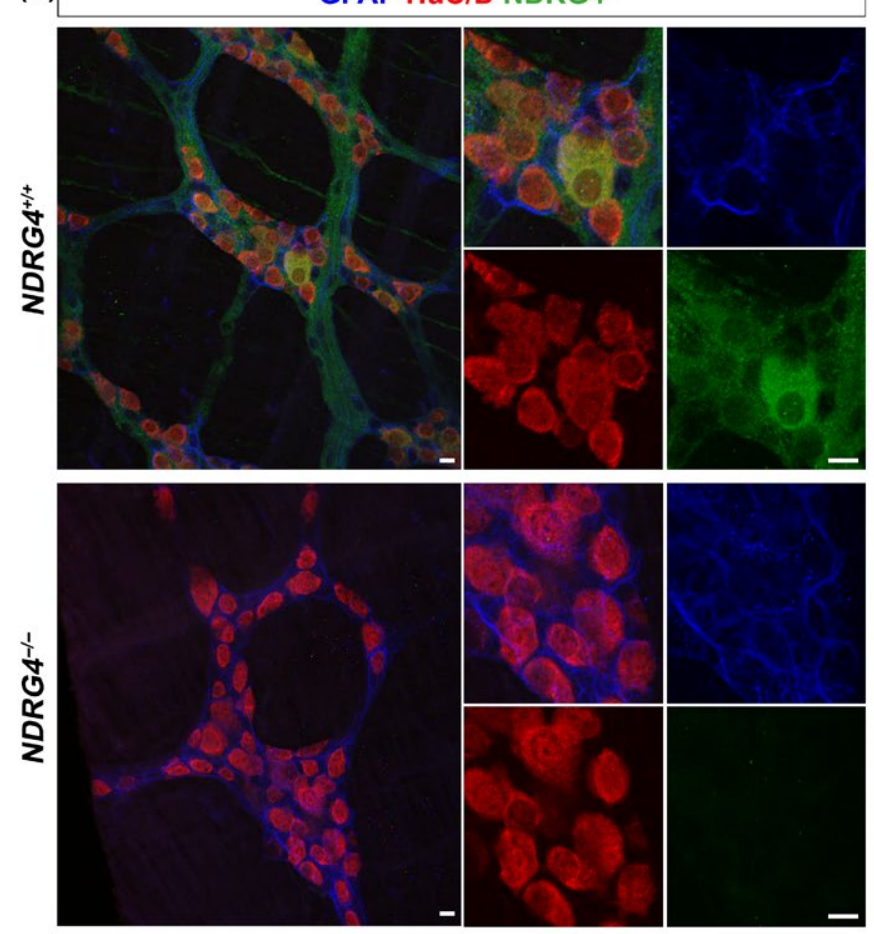

(C)

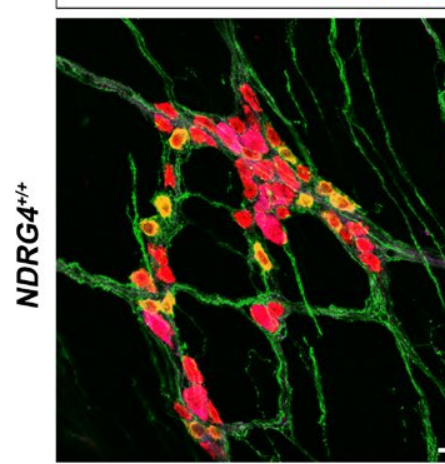

NDRG4 HuC/D nNOS

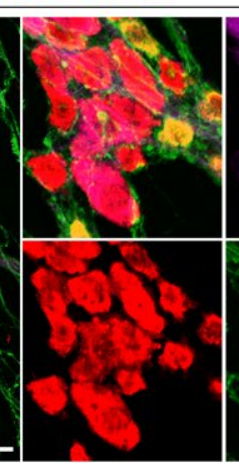

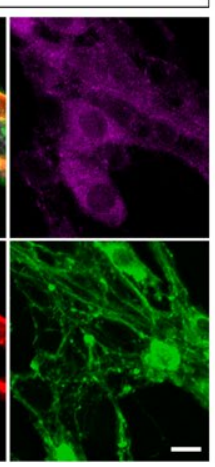

(B)
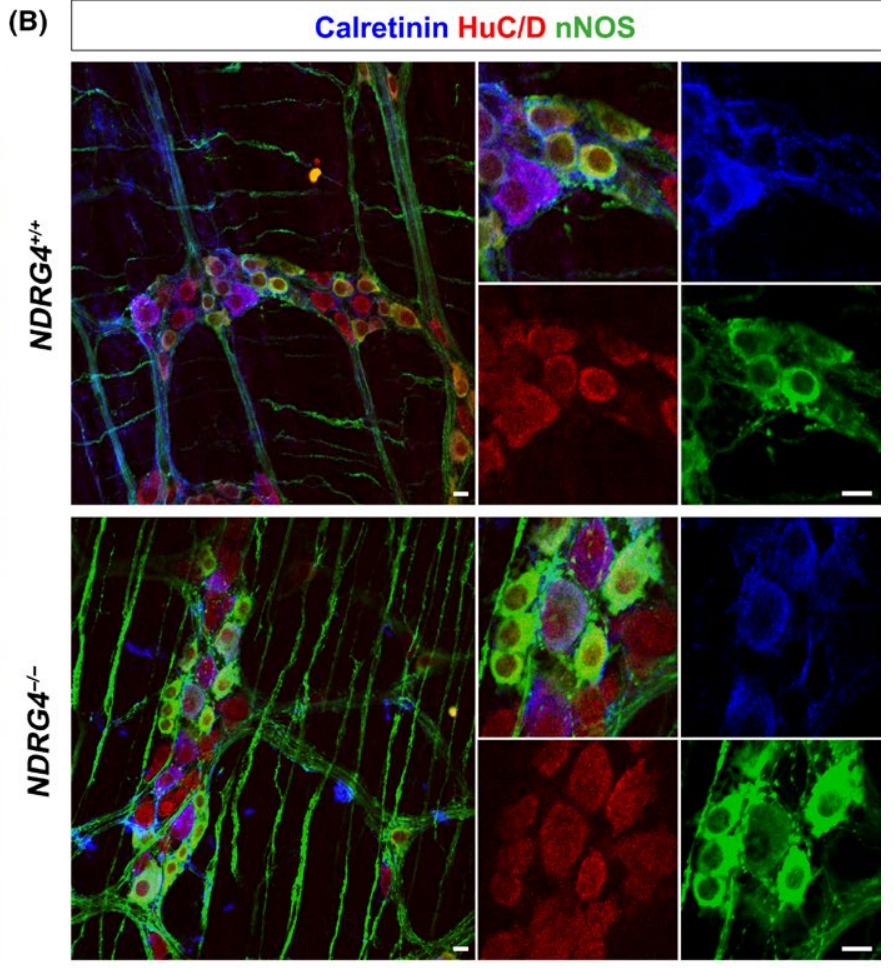

(D)

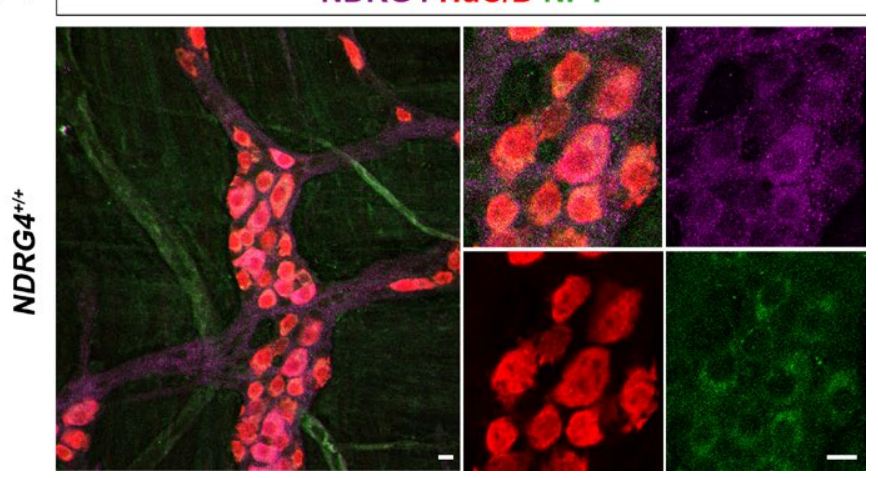

FIGURE 3 NDRG4 is expressed in different subsets of enteric neurons. (A-D) Representative fluorescently labeled whole-mount preparations of colonic myenteric plexus of NDRG4 $4^{+/+}$and NDRG4 ${ }^{-/-}$mice. Scale bars, $5 \mu \mathrm{m}$. A, NDRG4 always co-localized with HuC/D (green vs red) but never with GFAP (green vs blue). NDRG4 ${ }^{-/-}$mice don't express NDRG4, but still possess enteric neurons (red) and glial cells (blue). B, Plexus of $\mathrm{NDRG}^{+/+}$and $\mathrm{NDRG} 4^{-/-}$revealed similarities in HuC/D, calretinin and nNOS positivity (red, blue and green, respectively). C, NDRG4 positive cells (purple) are almost never reactive to nNOS (green) and nNOS positive cells almost never express NDRG4. D, NDRG4 (purple) co-localized with NPY (green) in various cell bodies of secretomotor neurons

Western blotting and immunohistochemical analysis using tissues of $\mathrm{NDRG}^{+/+}$and $\mathrm{NDRG}^{-/-}$mice revealed that both the Abnova and Cell Signaling antibody could discriminate all three known human NDRG4 isoforms. However, the specificity for NDRG4 in NDRG4 ${ }^{-/-}$ mice was only confirmed using the Cell Signaling antibody. Given the high sequence similarity (52\%-65\%) between NDRG4 and its protein family members, it is likely that the Abnova antibody recognizes one of the other NDRG proteins. According to the phylogenetic tree, NDRG4 shares the closest relation with NDRG2, but either NDRG1, 2 or 3 might be detected, as their main isoforms are 43,39 , or 41 and 40 or $41,5 \mathrm{kDa}$ in size respectively. ${ }^{1,4-7,10}$ Hence, this finding casts doubt on previously published statements that are based on the expression of NDRG4.
Using the validated Cell Signaling antibody, we explored the expression of NDRG4 throughout the body. We confirmed that NDRG4 is specifically expressed within neurons of the central nervous system (ie, brain and spinal cord) ${ }^{7,9}$ and observed this neuronal-specific expression pattern through the whole-body. More depth investigation of the expression pattern of NDRG4 within the gut revealed the presence of the NDRG4B ${ }^{\mathrm{var}}$ and NDRG4H isoform in murine colon and the specific expression of NDRG4 within the ENS. The ENS, the so-called brain of the gut, is an interconnected network of enteric neurons and glial cells, predominantly clustered in ganglia of the submucosal and myenteric plexuses, along the entire GI tract. ${ }^{24}$ Interestingly, NDRG4 has already been identified with DNA microarray analysis 


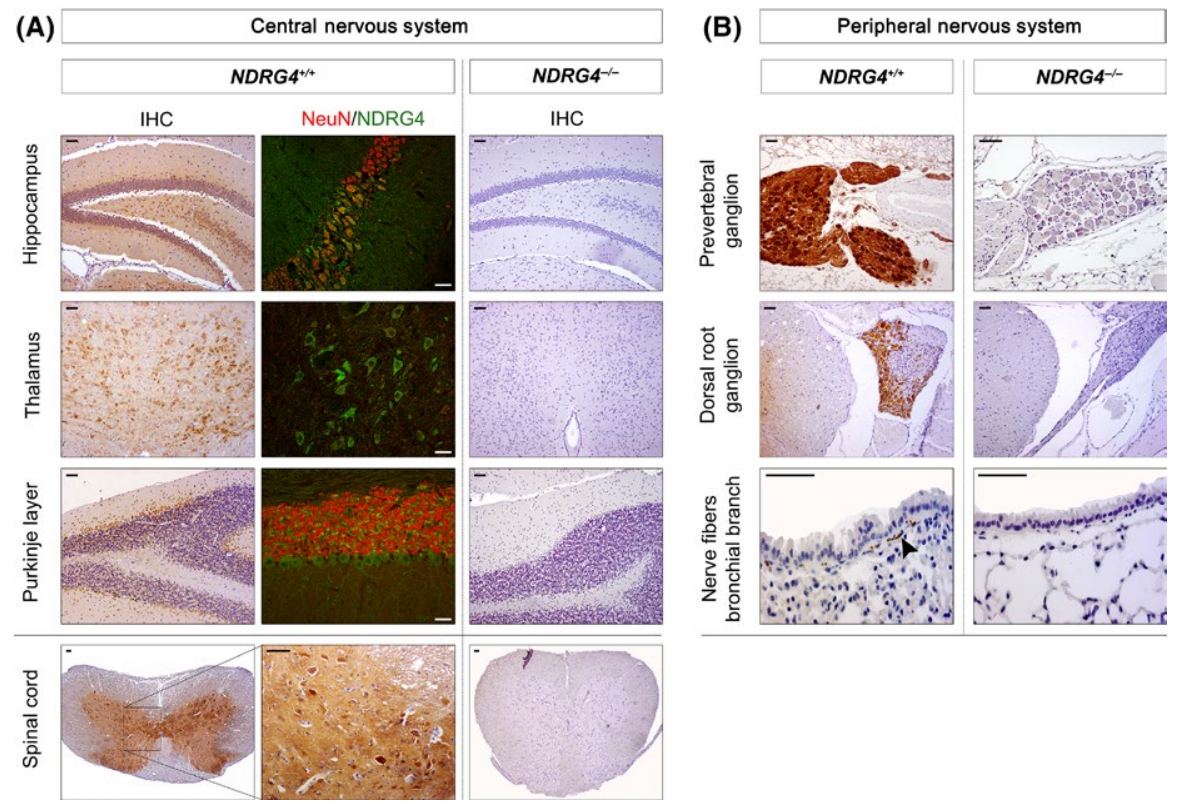

FIGURE 4 NDRG4 is specifically expressed in the central en peripheral nervous system. (A) Immunohistochemical and immunofluorescent analysis of several areas of the one-year old mouse brain. Gray matter structures in the cerebrum and cerebellum of NDRG4 ${ }^{+/+}$mice are positive for NDRG4 (green) and various regions show co-localization of NDRG4 with the neuronal marker NeuN (red): e.g. NeuN positive cells in the hippocampal cortex (pyramidal neurons and granule cells) and in the thalamus co-express NDRG4. Cerebral Purkinje cells, which are negative for NeuN, are strongly positive for NDRG4. The gray matter of the spinal cord also showed NDRG4 immunoreactivity within neuronal cell bodies (brown). NDRG4 is absent in all central nervous system structures in NDRG4 ${ }^{-1-}$ mice. (B) Representative images of peripheral tissues displayed NDRG4 expression in neurons, in prevertebral and dorsal root ganglia, and in nerves to, from and within every organ of the body (e.g. nerve fibers bronchial branch). White scale bars, $20 \mu \mathrm{m}$; and black scale bars, $50 \mu \mathrm{m}$ in A and B.

and in situ hybridization as one of the significantly down-regulated genes in the aganglionic bowel of mice (Ret $\left.{ }^{k-1 k^{-}}\right)$and Hirschsprung's patients. ${ }^{25,26}$ Though, here we describe for the first time that NDRG4 expression in the gut is restricted to enteric neurons as NDRG4 always co-localized with the pan-neuronal marker HuC/D, but never with the enteric glial cell marker GFAP. Together, our findings raise the question whether there is a role for the ENS in the development and/or progression of CRC.

While the reciprocal cross-talk between nerves and tumor cells, together with the concept of neoneurogenesis/axonogenesis ${ }^{27}$ is nowadays a rapidly evolving field, very little research has been conducted to unravel the role of the ENS in CRC development/ progression. Nevertheless, the importance of the ENS is emphasized by the broad range of (life-threatening) enteric neuropathies like Hirschsprung's disease that arise when alterations in the ENS occur. Furthermore, it has been described that impairments in the ENS can contribute to the development and severity of intestinal inflammation ${ }^{28}$ and that inflammatory conditions of the gut are often associated with changes in the ENS. ${ }^{29,30}$ A growing body of evidence demonstrates that proper functioning of the ENS, ensured by the interplay between enteric neurons and enteric glial cells, is pivotal to maintain the integrity of the intestinal epithelial barrier to regulate gut homeostasis. Neurons, like enteroendocrine cells, synthesize various neurotrophic factors and messenger molecules, which are packaged in vesicles and released in exocytotic processes. The process of exocytosis allows a cell to communicate with neighboring cells, ie, other neurons, glia, muscle, endothelial, immune and epithelial cells. ${ }^{22}$ It is well-established that several (enteric) neurotransmitters, eg, acetylcholine, vasoactive intestinal peptide and substance $P$, can target intestinal stem cells. ${ }^{31}$ These data suggest a potential role for the ENS in the development/ progression of CRC.

NDRG4 has been implicated in the route of vesicular transport. The interaction of NDRG4 with Blood vessel epicardial substance (Bves) has been shown to regulate docking of VAMP-3 (SNARE-protein) positive vesicles to the cell surface and the subsequent cargo delivery (eg, fibronectin). ${ }^{32}$ Similarly, NDRG4 controls vesicle membrane fusion during exocytosis as NDRG4 knockdown has been associated with a sharp reduction in the level of another vesicle-SNARE protein, SNAP25. ${ }^{12}$ The SNARE proteins, including SNAP25, are complexes that are essential in promoting subcellular trafficking, vesicular fusion and subsequent exocytotic release of neurotransmitters. ${ }^{33}$ Hence, it is reasonable that NDRG4, like NDRG1 and NDRG2, is able to modulate subcellular vesicle trafficking and exocytotic release of neuromediators, ${ }^{34,35}$ thereby regulating neurotransmission and the subsequent targeting of the epithelial layer.

In summary, we previously observed that the recognition of NDRG4 promoter methylation in fecal DNA is a valuable tool for the non-invasive detection of CRC and we identified NDRG4 as a tumor suppressor gene in CRC whose epithelial expression decreases from normal mucosa to CRC. However, using the validated Cell Signaling antibody, we found that NDRG4 is specifically expressed within central, peripheral and enteric neurons but not in epithelial cells. Although it still remains to be elucidated how the expression pattern of NDRG4 is linked with its biomarker performance for CRC, validating our 
hypothesis that NDRG4 can influence CRC development and/or progression via the ENS will have a major impact on current concepts in CRC research.

\section{DISCLOSURE}

The authors disclose no conflicts.

\section{AUTHOR CONTRIBUTION}

NV contributed to the literature search, study concept and design, data collection, data analysis and data interpretation, generation of figures and writing of the manuscript; MHFML contributed to the literature search, histological data collection and interpretation, and writing of the manuscript; MJG contributed to histological data collection, analysis and interpretation, and critical revision of the manuscript; GR contributed towards critical revision of the manuscript; KLD contributed to data collection; WB contributed to data collection and generation of figures; KADW contributed to data collection and interpretation; AG contributed towards data collection and generation of figures; $\mathrm{XQ}$ contributed towards critical revision of the manuscript; HPJS contributed towards data collection and interpretation; BPFR contributed towards critical revision of the manuscript; SHB contributed to data analysis and interpretation and critical revision of the manuscript; KAS, RMWH and MvE contributed towards data analysis and interpretation and critical revision of the manuscript; PvB contributed to data collection, data analysis and interpretation, generation of figures and critical revision of the manuscript; VM obtained funding and contributed to study concept and design, data analysis and data interpretation, and writing of the manuscript.

\section{REFERENCES}

1. Melotte V, Lentjes MH, van den Bosch SM, et al. N-Myc downstreamregulated gene 4 (NDRG4): a candidate tumor suppressor gene and potential biomarker for colorectal cancer. J Natl Cancer Inst. 2009;101:916-927.

2. Ahlquist DA, Zou $H$, Domanico $M$, et al. Next-generation stool DNA test accurately detects colorectal cancer and large adenomas. Gastroenterology 2012;142:248-256.

3. Imperiale TF, Ransohoff DF, Itzkowitz $\mathrm{SH}$, et al. Multitarget stool DNA testing for colorectal-cancer screening. N Engl J Med. 2014;370:1287-1297.

4. Melotte V, Qu X, Ongenaert M, et al. The N-myc downstream regulated gene (NDRG) family: diverse functions, multiple applications. FASEB J. 2010;24:4153-4166.

5. Zhou RH, Kokame K, Tsukamoto Y, Yutani C, Kato H, Miyata T. Characterization of the human NDRG gene family: a newly identified member, NDRG4, is specifically expressed in brain and heart. Genomics. 2001;73:86-97.

6. Qu X, Zhai Y, Wei H, et al. Characterization and expression of three novel differentiation-related genes belong to the human NDRG gene family. Mol Cell Biochem. 2002;229:35-44.

7. Yamamoto H, Kokame K, Okuda T, Nakajo Y, Yanamoto H, Miyata T. NDRG4 protein-deficient mice exhibit spatial learning deficits and vulnerabilities to cerebral ischemia. J Biol Chem. 2011;286:26158-26165.
8. Nakada N, Hongo S, Ohki T, Maeda A, Takeda M. Molecular characterization of NDRG4/Bdm1 protein isoforms that are differentially regulated during rat brain development. Brain Res Dev Brain Res. 2002;135:45-53.

9. Okuda T, Kokame K, Miyata T. Differential expression patterns of NDRG family proteins in the central nervous system. J Histochem Cytochem. 2008;56:175-182.

10. Qu X, Jia H, Garrity DM, et al. Ndrg4 is required for normal myocyte proliferation during early cardiac development in zebrafish. Dev Biol. 2008;317:486-496.

11. Ohki T, Hongo S, Nakada N, Maeda A, Takeda M. Inhibition of neurite outgrowth by reduced level of NDRG4 protein in antisense transfected PC12 cells. Brain Res Dev Brain Res. 2002;135:55-63.

12. Fontenas L, De Santis F, Di Donato V, et al. Neuronal Ndrg4 is essential for nodes of Ranvier organization in zebrafish. PLoS Genet. 2016;12:e1006459.

13. Dupays L, Kotecha S, Angst B, Mohun TJ. Tbx2 misexpression impairs deployment of second heart field derived progenitor cells to the arterial pole of the embryonic heart. Dev Biol. 2009;333:121-131.

14. Newton-Cheh $\mathrm{C}$, Eijgelsheim M, Rice KM, et al. Common variants at ten loci influence QT interval duration in the QTGEN Study. Nat Genet. 2009;41:399-406.

15. Pradidarcheep W, Labruyere WT, Dabhoiwala NF, Lamers WH. Lack of specificity of commercially available antisera: better specifications needed. J Histochem Cytochem. 2008;56:1099-1111.

16. Qu X, Li J, Baldwin HS. Postnatal lethality and abnormal development of foregut and spleen in Ndrg4 mutant mice. Biochem Biophys Res Commun. 2016;470:613-619.

17. Herdewyn S, Cirillo C, Van Den Bosch L, et al. Prevention of intestinal obstruction reveals progressive neurodegeneration in mutant TDP-43 (A315T) mice. Mol Neurodegener. 2014;9:24.

18. Vaes N, Gijbels MJ, Lentjes M, et al. Expression of NDRG4 in the mouse and human gastrointestinal tract is restricted to enteric neurons. Neurogastroenterology and Motility. 2016;28:56.

19. Zheng J, Li Y, Zhu S, et al. NDRG4 stratifies the prognostic value of body mass index in colorectal cancer. Oncotarget. 2016;7:1311-1322.

20. Chu D, Zhang Z, Zhou Y, et al. NDRG4, a novel candidate tumor suppressor, is a predictor of overall survival of colorectal cancer patients. Oncotarget. 2015;6:7584-7596.

21. Yang Q, Gu Y, Zhang X, et al. Uterine expression of NDRG4 is induced by estrogen and up-regulated during embryo implantation process in mice. PLOS ONE. 2016;11:e0155491.

22. Kolodziej MA, Weischer C, Reinges MH, et al. NDRG2 and NDRG4 expression is altered in glioblastoma and influences survival in patients with MGMT-methylated tumors. Anticancer Res. 2016;36:887-897.

23. Xing $\mathrm{Y}$, Tang B, Zhu C, et al. N-myc downstream-regulated gene 4 , up-regulated by tumor necrosis factor-alpha and nuclear factor kappa $\mathrm{B}$, aggravates cardiac ischemia/reperfusion injury by inhibiting reperfusion injury salvage kinase pathway. Basic Res Cardiol. 2016;111:11.

24. Furness JB. The enteric nervous system and neurogastroenterology. Nat Rev Gastroenterol Hepatol. 2012;9:286-294.

25. Heanue TA, Pachnis V. Expression profiling the developing mammalian enteric nervous system identifies marker and candidate Hirschsprung disease genes. Proc Natl Acad Sci U S A. 2006;103:6919-6924.

26. Vohra BP, Tsuji K, Nagashimada M, et al. Differential gene expression and functional analysis implicate novel mechanisms in enteric nervous system precursor migration and neuritogenesis. Dev Biol. 2006;298:259-271.

27. Magnon C, Hall SJ, Lin J, et al. Autonomic nerve development contributes to prostate cancer progression. Science. 2013;341:1236361.

28. Margolis KG, Stevanovic K, Karamooz N, et al. Enteric neuronal density contributes to the severity of intestinal inflammation. Gastroenterology 2011;141:588-598.

29. Villanacci V, Bassotti G, Nascimbeni R, et al. Enteric nervous system abnormalities in inflammatory bowel diseases. Neurogastroenterol Motil. 2008:20:1009-1016. 
30. Bernardini N, Segnani C, Ippolito C, et al. Immunohistochemical analysis of myenteric ganglia and interstitial cells of Cajal in ulcerative colitis. J Cell Mol Med. 2012;16:318-327.

31. Neunlist M, Van Landeghem L, Mahe MM, Derkinderen P, des Varannes SB, Rolli-Derkinderen M. The digestive neuronal-glial-epithelial unit: a new actor in gut health and disease. Nat Rev Gastroenterol Hepatol. 2013;10:90-100.

32. Benesh EC, Miller PM, Pfaltzgraff ER, et al. Bves and NDRG4 regulate directional epicardial cell migration through autocrine extracellular matrix deposition. Mol Biol Cell. 2013;24:3496-3510.

33. Tafoya LC, Mameli M, Miyashita T, Guzowski JF, Valenzuela CF, Wilson MC. Expression and function of SNAP-25 as a universal SNARE component in GABAergic neurons. I Neurosci. 2006;26:7826-7838.

34. Choi SC, Kim KD, Kim JT, et al. NDRG2 is one of novel intrinsic factors for regulation of IL-10 production in human myeloid cell. Biochem Biophys Res Commun. 2010;396:684-690.
35. Kachhap SK, Faith D, Qian DZ, et al. The N-Myc down regulated Gene1 (NDRG1) Is a Rab4a effector involved in vesicular recycling of E-cadherin. PLOS ONE. 2007;2:e844.

\section{SUPPORTING INFORMATION}

Additional Supporting Information may be found online in the supporting information tab for this article.

How to cite this article: Vaes N, Lentjes MHFM, Gijbels MJ, et al. NDRG4, an early detection marker for colorectal cancer, is specifically expressed in enteric neurons. Neurogastroenterol Motil. 2017;00:e13095. https://doi.org/10.1111/nmo.13095 